\title{
A Hubble Instrument Comes Home: UW-Madison's High Speed Photometer
}

\author{
James M. Lattis ${ }^{1}$ \\ Director, UW Space Place \\ Department of Astronomy, University of Wisconsin-Madison
}

\begin{abstract}
WSGC provided generous support to return the High Speed Photometer (HSP) to Wisconsin to be used for public education and outreach at UW Space Place. HSP was one of the original five science instruments built for and launched with the Hubble Space Telescope (HST). Declared to be federal surplus equipment in autumn 2011, HSP was acquired by UW Space Place and brought to Madison to be put on exhibit and used for public education. As the only research photometer built specifically for HST, HSP is an important part of the history of astronomical instrumentation and one of the most important astronomical instruments to come from Wisconsin.
\end{abstract}

\section{Introduction}

UW Space Place requested and was granted support from Wisconsin Space Grant Consortium for the relocation of a scientific instrument that is very significant in the history of astronomical research and a primary example of Wisconsin's contributions to space science. The High Speed Photometer project was a direct descendant of the continuous development of astronomical photoelectric photometry dating to the early work of Prof. Joel Stebbins, Director of Washburn Observatory from 1922 to 1948. Stebbins and his younger colleague Prof. Albert Whitford advanced photoelectric photometry to such an extent that their practices and instruments were widely adopted as standards in the astronomical community.

With the coming of the Space Age, the capacity to put astronomical instruments in orbit led to NASA's Orbiting Astronomical Observatory-2, which was the first general purpose space observatory and the first to carry out extended observations in the ultraviolet. The principal and most productive instruments aboard OAO-2 were the photometers of the Wisconsin Experiment Package (WEP), which was designed and operated by the UW Space Astronomy Laboratory (SAL).

\section{Origins of HSP}

The technical and scientific success of WEP aboard OAO-2 ensured that Wisconsin's astronomers were deeply involved in the planning for the Space Telescope (later Hubble Space Telescope) project in its earliest stages. The Space Telescope was conceived as a general purpose, primarily optical, observatory in Earth orbit that could be serviced by astronauts but operated by astronomers from the ground. With photoelectric photometry by then a proven technique for space observations, Prof. Robert Bless, who had been one of the leading OAO-2 team members, proposed a minimalist photometric instrument as part of the new space observatory. The instrument, which he characterized as "two thermos bottles and a shoebox" (meaning two photometer tubes and their associated electronics), would be relatively small, simple, and inexpensive compared to the other much more complex "science instruments," or SIs, to be included in the Space Telescope.

1 UW Space Place wishes to acknowledge the support of the Wisconsin Space Grant Consortium in the acquisition of HSP. We are also grateful for support from: Judy \& Jim Sloan Foundation, Prof. Robert C. Bless, UW-Madison Space Science \& Engineering Center, UW-Madison Department of Astronomy. 
The motivation for a space-based general purpose photometer was three-fold: First, it could extend optical ground-based photometry into the ultraviolet, as OAO-2 had done, but with the vastly greater power offered by a large telescope. Second, it could perform polarimetry, a very powerful technique for investigating the characteristics of interstellar matter and complex star systems, for example. Third, freed of the scintillation of Earth's atmosphere, a space-based photometer can make very high time-resolution studies, i.e. analyzing changes in the light from an object millisecond by millisecond or better. This time-resolution is useful, for example, with rapidly fluctuating objects, like pulsars, and explosive events, such as recurrent novas. This ability to take up to 100,000 measurements per second gave the Wisconsin instrument its appellation as the "high speed" photometer.

NASA considered the scientific case for a photometer aboard the space observatory to be so compelling as to solicit from UW a proposal for a full, major Science Instrument (of which there can be five at any given time) devoted to high-speed photometry. For the full proposal, Bless and his team at SAL collaborated with UW's Space Science and Engineering Center. The result was a contract to deliver a High Speed Photometer in 1983 that would be one of the five original science instruments (SIs) aboard the Hubble Space Telescope when it was launched into orbit.

Design, fabrication, and testing of HSP was a large step in complexity beyond anything undertaken by UW astronomers up to that point. Aside from some NASA-supplied standard hardware and off-the-shelf aerospace equipment (such as power supplies) available from commercial sources, the entire instrument, including structural and thermal design, detectors and their support electronics, on-board control systems, scientific and control software, and groundbased testing procedures, were developed and fabricated entirely at UW-Madison. HSP was the only HST SI to originate entirely on a university campus, and the SAL/SSEC team also made extensive use of student employees, both graduate and undergraduate. The instrument was delivered on schedule and under budget and passed all NASA verification and acceptance tests without significant problems.

HSP was also unique among HST SIs in the simplicity and reliability of its design, which allowed hundreds of filter-aperture-detector configurations with no moving parts, "except electrons," as Prof. Bless says. This elegant simplicity was made possible by the use of Image Dissector Tubes (IDTs) as the primary detectors. IDTs were the direct descendants of the photoelectric photometers that had been developed by UW astronomers since the 1920s and which were the most advanced form reached by photoelectric detectors before the advent of solid-state detectors, by which IDTs have now been replaced. For that reason, HSP (along with its sibling instrument, the Wisconsin Ultraviolet Photo-Polarimeter Experiment) represents the historical capstone and most advanced form of the astronomical photometry that Wisconsin astronomers had pioneered for most of the Twentieth Century.

\section{Mission}

HSP was delivered to NASA as contracted in 1983, but numerous problems in NASA's HST and shuttle programs resulted in repeated launch delays. In the end, HSP and the other original SIs were launched in place with HST in 1990. As is well known, HST began operations with several serious technical problems. Foremost, and best known, is that Perkin-Elmer Corp. made a mirror 
with significant spherical aberration, and that fundamental optical error was not caught in any NASA acceptance testing. The result is that the prime focus images are blurry, e.g. the images of stars that should be diffraction-limited points are instead much larger, fuzzy spots. Another major problem came in the telescope's pointing stability - far worse than the specificationswhich meant that those fuzzy images could not be kept steady in the field of view.

These two problems were devastating for the scientific potential of all of the SIs. In the case of HSP, the pin-point images produced by HST's advertised optical perfection were supposed to direct starlight through tiny apertures in front of the IDTs. Instead, the fuzzy images were larger than most of the apertures, so much of the incoming starlight never reached the detectors. The pointing instability meant that even those fuzzy images could not be depended upon to remain centered on a given aperture.

Despite these problems, Bless's team of astronomers were able to carry out a number of new observations and produce meaningful results. (A bibliography of the refereed publications based on HSP science can be found at http://www.sal.wisc.edu/HSP/hsp.papers.html.) Examples of HSP's scientific results include:

- Highest time-resolution optical light curve, and the first ever ultraviolet light curve, of the Crab Nebula pulsar.

- Detailed structural investigations of Saturn's rings by means of stellar occultation.

- First high time-resolution ultraviolet photometry over the complete eruption cycle of a recurrent nova.

- Ultraviolet polarimetry of galaxy images to test theories of gravitational lensing.

HST's pointing instability was found to be largely a result of thermal instability and mechanical flexure in the solar panels. This was solved during the first HST servicing mission, in December 1993, when the original solar panels were replaced with a new design. Correction of the spherical aberration problem was accomplished by installation of a device designed to take the place of one of the primary science instruments in order to insert corrective optics in front of the remaining SIs. (This was often described as giving HST "eyeglasses," although more accurately it amounted to giving each SI its own set of "eyeglasses.") In order to install this corrective optics device, called COSTAR (for Corrective Optics Space Telescope Axial Replacement), as part of the servicing mission, one of the original SIs had to be removed, and NASA judged that HSP would be the odd instrument out. So HSP was removed from HST by astronauts, who then installed COSTAR in its place. As is well known, COSTAR, along with the solar panel replacement, succeeded in bringing HST up to specifications and restored the remaining instruments to their full potential. Thus the sacrifice of HSP saved the mission for the other instruments, but at the cost of all the potential science that HSP's team could have accomplished. Throughout its operational life, HSP operated flawlessly.

\section{Denouement}

HSP was returned from orbit at the end of the servicing mission. Except for a brief return to Madison for post-flight calibration tests, the instrument remained in a NASA storage facility until autumn of 2011. At that time, following the end of the shuttle program (and hence the end of any possibility of further HST servicing missions), HSP was declared federal surplus equipment. UW Space Place thereupon requested and was granted ownership of HSP for 
purposes of public exhibition and education. The not insignificant shipping costs of such a transfer must be borne by the receiver. We requested and were granted funding by Wisconsin Space Grant Consortium as partial support for shipping HSP to Madison.

Since its arrival in Madison, and with support from UW-Madison's SSEC and Astronomy Dept., HSP has been mounted on a custom dolly to make it practical and safe for public exhibition. Some of the aluminum skins have been replaced by plexiglass panels so that visitors can see the internal structure, components, and instrumentation within. HSP went on public display at UW Space Place on 20 July 2012 and will be maintained there as a permanent exhibit (Fig.1).

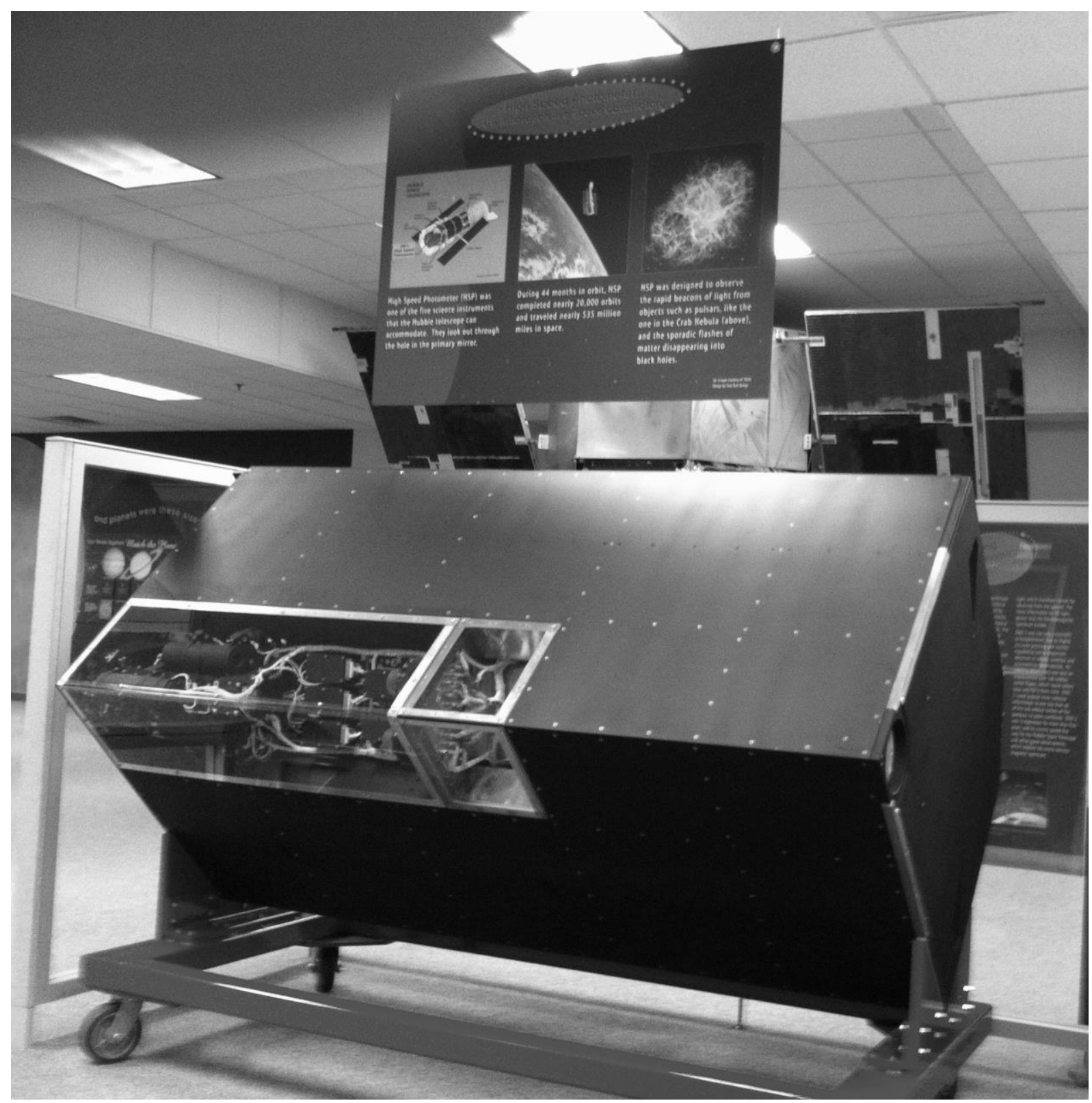

Fig 1. HSP on public display at UW Space Place 